\title{
Importancia de la entrevista médico forense en la evaluación del abuso sexual en el paciente pediátrico
}

Grettchen Flores-Sandí

Resumen

Justificación y objetivo: Aún en casos confirmados legalmente de abuso sexual infantil, la revelación del niño es a menudo la pieza más importante de información en la determinación del probable abuso. En el peritaje médico forense de delitos sexuales en niños, existen aspectos diferenciales en el reconocimiento clínico y reglas que se deben cumplir y que pueden impactar la calidad de la valoración. Analizar la importancia de la entrevista médico forense en la evaluación del abuso sexual en el paciente pediátrico.

Metodología: Se realizó un estudio prospectivo a una muestra aleatoria de 53 niños menores de 12 años, atendidos durante el primer semestre de 2006, en la Clínica Médico Forense del Departamento de Medicina Legal en Costa Rica. Las variables estudiadas fueron motivo de consulta, edad, nacionalidad, escolaridad, su acompañante durante la valoración, fuente de información (directa o indirecta), tipo de contacto o abuso que refirieron, antecedentes patológicos, anuencia o renuencia a colaborar, lesiones documentadas e incapacidad temporal o permanente.

Resultados: Con respecto al motivo de denuncia, 49 casos correspondieron a abuso sexual, 3 a violación y uno a corrupción de menores el $79 \%$ fueron niñas. Solamente un caso no era costarricense y en cuatro casos la historia fue indirecta. El rango de edades estuvo entre los 2 y los 12 años, 25 menores de seis años, y 40 acudieron acompañados por la madre. 36 casos reportaron que el tipo de contacto fue digital y solamente en siete casos se documentó algún antecedente de importancia. Solo en dos casos se presentaron lesiones genitales, otros dos casos presentaron contusiones simples en el nivel extragenital, y en tres casos se encontró eritema genital como hallazgo inespecífico. Todos los menores colaboraron con la realización del examen físico y ninguno de ellos ameritó alguna incapacidad temporal ni permanente.

Conclusión: La parte vital de toda la valoración completa en estos casos es la experiencia del médico examinador, quien se sugiere que deba transmitirles ciertas cualidades de actitud para lograr la colaboración de los menores.

Descriptores: abuso sexual infantil, entrevista médica, evaluación clínica forense.

Key words: childhood sexual abuse, medical interview, clinical forensic evaluation.

Recibido: 16 de agosto de 2006 Aceptado: 24 de marzo de 2007

Departamento Medicina Legal, Poder Judicial.

Correspondencia:

Correo electrónico:gflores68@

racsa.co.cr

ISSN 0001-6002/2007/49/3/149-153 Acta Médica Costarricense, $\mathbb{C} 2007$

Colegio de Médicos y Cirujanos
La definición de abuso sexual infantil se establece como el involucramiento de niños en actividades sexuales que ellos no comprenden verdaderamente, de las cuales no son capaces de otorgar un consentimiento informado. La actividad sexual puede incluir caricias, contacto oralgenital, genital y anal, así como exhibicionismo, voyerismo y exposición a la pornografía. El abuso sexual debe diferenciarse del "juego sexual" (comportamiento apropiado para la edad"), 
donde el nivel de desarrollo de los participantes es similar y ocurre sin coerción. Por ejemplo, niños preescolares viéndose los genitales mutuamente es considerado como normal, mientras que se requiere investigación si un niño más maduro (o no necesariamente) en su desarrollo, forza a uno menor con comportamientos sexuales.

El examen físico para probar que un delito sexual ha ocurrido ha sido una de las tareas medulares en la práctica médico legal desde el inicio de la medicina forense clínica. Las medidas diagnósticas incluyen la valoración de los hallazgos genitales, la documentación de traumas extragenitales y la evaluación de evidencias. ${ }^{2}$

Contrario a lo que las autoridades esperarían, solo un porcentaje comparativamente pequeño de víctimas de delitos sexuales muestran traumas genitales, aún en casos confirmados de abuso sexual. ${ }^{2,3}$ Se describen lesiones en menos del $10 \%$ de los niños que sufrieron abuso. ${ }^{4}$

Por lo tanto, la revelación del niño es a menudo la pieza más importante de información para determinar el probable abuso. ${ }^{1,5,6}$

Sin embargo, el hecho de que la entrevista no puede subestimarse, lo entienden mejor los profesionales en derecho que en medicina. En este sentido, Christian y colaboradores indican que el grado de entrenamiento y la experiencia del médico que atiende a los niños pueden impactar la calidad de la valoración. ${ }^{7}$ Por su edad, existen aspectos diferenciales en el reconocimiento clínico y reglas que se deben cumplir en la conversación con estos.

El objetivo del presente trabajo es analizar la importancia de la entrevista médico forense en la evaluación del abuso sexual en el paciente pediátrico.

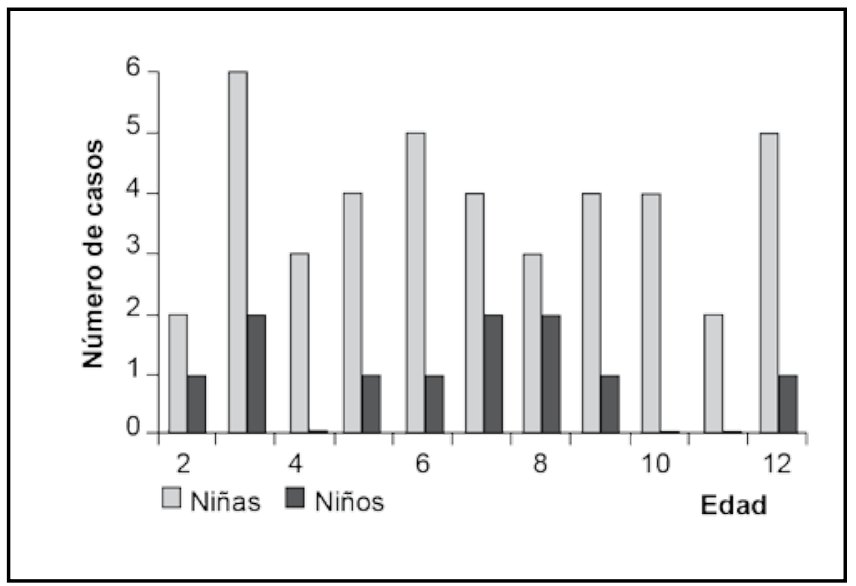

Figura 1. Distribución según edad y sexo, de una muestra de menores atendidos en la Clínica Médico Forense durante el I semestre de 2006

\section{Metodología}

Se realizó un estudio prospectivo en una muestra de 84 pacientes menores de 12 años atendidos durante el primer semestre de 2006, en la Clínica Médico Forense del Departamento de Medicina Legal en Costa Rica, obtenida mediante muestreo aleatorio simple. Se obtuvo que 53 de ellos $(63 \%)$ correspondieron a denuncias de delitos sexuales, de los cuales 42 resultaron ser niñas y 11 varones. El método clínico como estudio de casos permitió conocer el motivo de consulta, edad de los menores, nacionalidad, escolaridad, acompañante durante la valoración y fuente de información (directa o indirecta) durante la valoración. También se buscó documentar el tipo de contacto o abuso que refirieron, los antecedentes patológicos de los niños, la anuencia o renuencia al examen físico, las lesiones que se documentaron y si alguno de ellos ameritó incapacidad temporal o permanente por alguna lesión. Todos los casos se abordaron considerando el respeto y empatía que deben primar en la relación médico-paciente.

\section{Resultados}

Con respecto al motivo de denuncia, 40 casos (75\%) correspondieron a abuso sexual, $12(23 \%)$ a violación y uno $(2 \%)$ a corrupción de menores. En cuanto a la nacionalidad, solamente un caso (una niña de 12 años de edad) no era costarricense, sino nicaragüense. Las edades de los niños estuvieron entre los 2 y los 12 años, la mayoría $(25 / 53 ; 47 \%)$ fueron menores de seis años y acudieron a la valoración acompañados por la madre $(40 / 53 ; 75 \%)$. Las figuras 1 y 2 , muestran la distribución de acuerdo con la edad y la escolaridad de los menores.

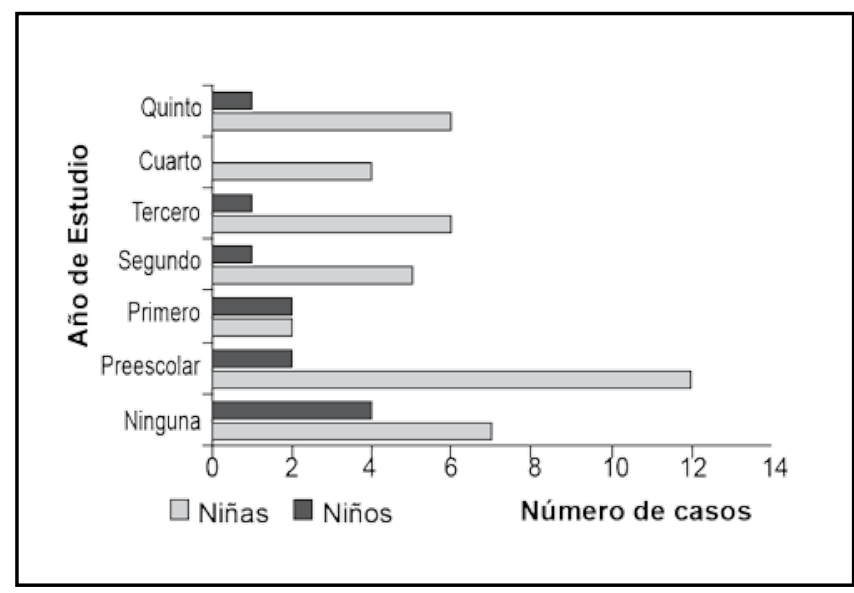

Figura 2. Distribución según nivel de escolaridad, de una muestra de menores atendidos en la Clínica Médico Forense durante el I semestre de 2006 
Tres niñas entre los cinco y los siete años, referidas por sospechas de abuso sexual, negaron cualquier tipo de este.

Los cuatro niños de quienes se obtuvo la historia de forma indirecta se encontraban en edad preescolar (2-3 años) y no asistían a ningún centro infantil, en comparación con niños de las mismas edades que asisten a estos centros y de quienes sí se obtuvo historias directas, lo que podría justificarse por el grado de socialización y desarrollo de vocabulario de los últimos. Todos los menores colaboraron con la realización del examen físico y ninguno de ellos ameritó alguna incapacidad temporal ni permanente. La mayoría de los menores (36 casos $/ 53 ; 68 \%$ ) reportó que el tipo de contacto fue digital, el contacto genital solo lo reportaron 20 menores (38\%), uno de ellos lo señaló como ocurrido en sus piernas (Cuadro 1).

Únicamente siete casos refirieron antecedentes patológicos, secuelas por luxación congénita de cadera con rigidez residual, microftalmia con prótesis ocular, fimosis, infección de tracto urinario a repetición y drogodependencia. Dos pacientes refirieron historia de asma en tratamiento y un antecedente de hernioplastía inguinal.

En dos casos se observaron lesiones genitales. En uno hubo rupturas de himen y en otro caso, en la horquilla vulvar. Dos pacientes presentaron contusiones simples en el nivel extragenital. En tres niños se encontró eritema genital como hallazgo inespecífico. En uno de los casos en el que el menor presentó contusiones simples en el nivel extragenital, se encontró un dibujo de representación fálica en uno de sus glúteos.

\section{Discusión}

El proceso de la entrevista médica en caso de abuso sexual de menores debe realizarse con cuidado, absteniendo a la víctima de repetir su historia en diferentes profesionales, pues esto podría hacerle reexperimentar el sufrimiento sufrido e intensificarlo. ${ }^{6,8}$ En Costa Rica generalmente la entrevista médico forense se realiza antes de la valoración psicológica, y en ocasiones los menores no han dado ninguna "declaración" a las autoridades judiciales al momento cuando sus padres o encargados establecieron la denuncia, lo cual aporta mayor relevancia a los datos que se obtengan de la historia médico legal, por la posibilidad de retractación y olvido.

Desde el punto de vista médico, la entrevista debe incluir información útil para determinar qué exámenes deben realizarse y cuándo, cómo interpretar hallazgos médicos presentes y qué servicios médicos y psiquiátricos deben recomendarse al niño y su familia. ${ }^{6}$ Además de explorar el padecimiento actual, la entrevista debe incluir las preguntas relevantes para obtener detalles de la historia pediátrica, la
Cuadro 1. Tipos de contacto reportados

\begin{tabular}{|l|l|c|}
\hline \multicolumn{2}{|l|}{ Tipo de contacto } & Número de casos \\
\hline Oral: & Genital & 2 \\
\hline Digital: & Vagina & 26 \\
& Pene & 3 \\
& Ano & 3 \\
& Cuerpo & 4 \\
\hline Genital: & Oral & 1 \\
& Vagina & 13 \\
& Ano & 5 \\
& Piernas & 1 \\
\hline Objeto: & Vagina & 2 \\
\hline Otros: & Exhibicionismo & 4 \\
& Dibujo fálico & 1 \\
\hline Niega & & 3 \\
\hline Desconoce & & 2 \\
\hline
\end{tabular}

historia médica y los antecedentes de abuso o traumas sospechosos, y en niñas y adolescentes postpúberes, debe documentarse la historia menstrual. Cuando los menores son llevados a valoración por personal de servicios de protección a las víctimas, solamente está disponible la historia que brinda el niño.

El niño debe ser entrevistado mediante preguntas abiertas, de preferencia sin su acompañante, en caso de que este distraiga su atención, genere influencia o dude de su historia frente al médico. Es importante mantener un tono neutral de voz y comportamiento cuando el niño responde y no hacer preguntas sugestivas, preguntar adelantándose a sus respuestas o mostrar emociones fuertes, tales como choque o incredulidad, y mantener un acercamiento "cuénteme más” o "y luego qué pasó”. 1,6,10

El uso de una serie de preguntas concretas (¿qué te hizo?, ¿con qué?, ¿dónde?), más que una pregunta simple, (han abusado sexualmente de usted?) es crítico, pues es probable que el niño no sepa lo que significa un abuso sexual. En este tipo de interacción, un niño puede aprender por primera vez que lo que vivió es considerado inapropiado y abusivo.

En general, realizar una entrevista clínica eficaz a un paciente pediátrico requiere motivación y destreza en el ejercicio profesional por parte de un médico. ${ }^{5,9,11}$ De acuerdo con Hetnecker, Forman y Daruna ${ }^{9}$, las conversaciones profesionales con niños están sujetas a ciertas reglas, las cuales bien pueden aplicarse a la valoración forense de pacientes pediátricos.

Hay que tener en cuenta la diferencia existente entre una exploración cuyo objetivo es realizar un diagnóstico para 
aplicar un tratamiento, y una valoración como esta, en la que el objeto del reconocimiento es valorar la presencia de lesiones. ${ }^{10}$ Se debe recordar que la entrevista médica puede ser admitida en la corte. El establecimiento de una adecuada relación médico-paciente es muy importante en estas situaciones, por lo que el médico debe encontrar, en cada caso, algún elemento o varios que la faciliten. ${ }^{12}$

Una vez que se ha establecido el contacto adecuado y antes de la exploración propiamente dicha, hay que explicarle al niño (siempre en función de su edad y de su capacidad de comprensión) y a su acompañante, qué es lo que se hará y para qué, de manera que se consiga un grado suficiente de colaboración. ${ }^{1,10}$

En la presente serie, el contacto genital del ofensor solo lo reportaron veinte menores, y en dos de los casos se documentaron lesiones. Como se ha mencionado, el abuso sexual raramente se diagnostica sobre la base exclusiva del examen físico o los hallazgos de laboratorio. Con frecuencia, los hallazgos físicos están ausentes; incluso cuando el ofensor admite la penetración de los genitales del niño, muchos tipos de abuso no dejan evidencia física, y los traumas de mucosas a menudo curan rápida $\mathrm{y}$ completamente.

En ocasiones los investigadores judiciales y los padres pueden mostrarse escépticos por la información del niño cuando esta reporta penetración y no se encuentran anormalidades. Se debe tener claro que la penetración es un concepto que los adultos entienden, pero los niños pequeños no. ${ }^{5}$ El grado de penetración en las áreas anal y genital y la posible curación de los traumas son factores importantes a considerar.

El examen físico de los niños que han sufrido abuso sexual tampoco debe resultar en trauma físico o emocional adicional. Se debe tener en cuenta que así como desde el comienzo de la entrevista se aprecian detalles que corresponden al examen clínico, es más fácil completar la anamnesis con el niño mientras es examinado.

Aunque la mayoría de hallazgos en caso de abuso sexual o violación son normales o inespecíficos, los hallazgos médicos positivos continúan siendo el factor más importante en las investigaciones de estos delitos. Una puntuación de lesiones clínicas fue diseñada por miembros del Sexual Assault Service ${ }^{13}$ en Vancouver, de acuerdo con la valoración clínica de los reportes médico-legales, tomando en cuenta la extensión del trauma (Cuadro 2).

Muy pocos hallazgos pueden ser considerados como pruebas; entre ellos la presencia de espermatozoides o embarazo, los traumas genitales no accidentales especialmente en la parte posterior de la vagina, himen y vestíbulo, desgarros recientes o cicatrices de himen, traumas analesnoaccidentales, exámenes positivos para enfermedades venéreas (sífilis, gonorrea, VIH), si se excluye una infección perinatal y durante el embarazo. ${ }^{3}$

\section{Cuadro 2: Puntuación de lesiones clínicas}

\begin{tabular}{|l|l|}
\hline Puntuación & Criterio \\
\hline 1 (sin lesión) & $\begin{array}{l}\text { No se documentan signos o síntomas de } \\
\text { lesión. }\end{array}$ \\
\hline 2 (lesión & $\begin{array}{l}\text { Eritema o dolor solamente o lesiones } \\
\text { menores en que no se espera impacto } \\
\text { sobre la función física. }\end{array}$ \\
\hline 3 (lesión grave) & $\begin{array}{l}\text { Lesión o lesiones que se espera que } \\
\text { tengan algún impacto en la función y } \\
\text { más que eritema o dolor de los genitales } \\
\text { (incluyendo áreas anal y rectal), por } \\
\text { ejemplo: laceraciones, equimosis, } \\
\text { excoriaciones, y lesiones que requieren } \\
\text { tratamiento (laceraciones que requieren } \\
\text { sutura, heridas que necesitan vendajes) y } \\
\text { contusiones en la cabeza y cuello que se } \\
\text { espera resulten en cefalea significativa. }\end{array}$ \\
\hline $\begin{array}{l}\text { Trauma en la cabeza con concusión o } \\
\text { evidencia de intento de estrangulación } \\
\text { y otras lesiones mayores, por ejemplo: } \\
\text { fractura, contusión de órganos internos. }\end{array}$ \\
\hline
\end{tabular}

Se debe tener presente que en toda valoración médica la obtención de la historia y el examen físico son parte de un continuo. Los niños pueden colaborar si se les ofrece un trato adecuado y justo a su nivel de desarrollo cognitivo. ${ }^{14}$ Por lo tanto, el grado de entrenamiento y la experiencia del médico forense, que atiende pacientes pediátricos con este tipo de denuncias, influyen en la calidad de la valoración.

Lograr el acceso a un niño es parte del reto que enfrenta diariamente el médico forense; en tanto es posible que un médico poco entrenado sugiera al niño respuestas, también lo es que le sugiera negarse a la valoración, lo que no contribuiría en nada al logro de los objetivos de la investigación y podría constituir un aspecto dilatorio del proceso judicial, exponiendo al niño y a otros, al riesgo de sufrir lesiones.

Si bien la mayoría de los casos de la muestra estudiada, tal y como lo reporta la bibliografía, no presento lesiones, o bien, estas fueron inespecíficas, todos los menores permitieron la valoración médica $\mathrm{y}$, casi la totalidad, (exceptuando dos niños y una niña entre los dos y los tres años de edad) dieron información directa sobre el tipo de contacto que sufrieron. La importancia de la entrevista llevada a cabo como parte de la evaluación médico forense radica precisamente en el hecho de que la mayoría de las veces es el único hallazgo positivo de que ha existido abuso, y permite orientar la toma de muestras y contribuir con la posterior valoración psicológica de la víctima. La parte vital de la valoración completa en estos casos es la experiencia del médico examinador, quien se sugiere que transmita además de la conducta de motivación, congruencia, credibilidad e integridad, ciertas cualidades de actitud para lograr la colaboración de los menores (Cuadro 3). 


\begin{tabular}{|c|c|}
\hline \multicolumn{2}{|c|}{$\begin{array}{l}\text { Cuadro 3. Cualidades de actitud que se } \\
\text { deben transmitir al menor }\end{array}$} \\
\hline Actitud & Forma \\
\hline Respeto & $\begin{array}{l}\text { Llamarlo por su nombre, evitar trato de } \\
\text { minusvalía o afecto. Hacerle sentir que el } \\
\text { médico le brinda un servicio. }\end{array}$ \\
\hline Confianza & $\begin{array}{l}\text { Dejarlo reconocer el consultorio, jugar y } \\
\text { preguntar sobre las cosas que ve. Si se } \\
\text { prefiere, que cierre él o ella el consultorio. }\end{array}$ \\
\hline Comprensión & $\begin{array}{l}\text { Aclararle que su visita es para determinar la } \\
\text { presencia o no de lesiones y el sinnúmero de } \\
\text { niños que acuden por asuntos similares a la } \\
\text { suya, lo que justifica la imposibilidad de que } \\
\text { el médico recuerde lo que diga. }\end{array}$ \\
\hline Cooperación & $\begin{array}{l}\text { Indicarle de acuerdo con su nivel cognitivo, } \\
\text { que por lo que le pasó, es importante } \\
\text { observarlo para saber que no está enfermo. } \\
\text { Y explicarle según su comprensión, en qué } \\
\text { consiste el examen físico. }\end{array}$ \\
\hline Valentía & $\begin{array}{l}\text { Indicarle lo importante de que haya hablado, } \\
\text { que es muy valiente, casi un héroe, para que } \\
\text { no se lo sigan haciendo ni haya más niños a } \\
\text { los que se lo hagan y luego se pueda sentir } \\
\text { culpable por callar. }\end{array}$ \\
\hline Oportunidad & $\begin{array}{l}\text { Manifestarle la importancia de ser valorado } \\
\text { esta vez y no posponer la valoración, pues } \\
\text { la tensión y vergüenza pueden resultar } \\
\text { mayores. }\end{array}$ \\
\hline
\end{tabular}

\section{Abstract}

Justification: Even in confirmed cases of childhood sexual abuse, the child's interview is often the most important element in its determination. During the pediatric sexual forensic analysis there are clinical differences and rules that must be followed and that can influence the outcome of the assessment

Objective: To analyze the importance of forensic interview in the evaluation of sexual abuse in pediatric patients.

Methodology: a randomized prospective study was conducted during the first semester of 2006, at the Forensic clinic of the Legal Medicine Department in Costa Rica. The variables analyzed were: cause of consultation, age, nationality, years of school, accompanying person during the evaluation, source of information (direct or indirect), type of abuse or contact, willingness or unwillingness to collaborate, the presence of obvious lesions and physical disabilities found.

Result: The sample was of 53 children. The reason of consultation in 40 cases was sexual abuse, in 12 it was rape and in 1 it was corruption of minors, $79 \%$ of the individuals were female. Only 1 person was not Costa Rican and in 4 cases the source of information was indirect. The ages were between 2 and 12 years old, $47 \%$ were below 6 years of age and 40 children were accompanied with their mothers. in 36 cases there was hand/finger contact and only in 7 cases there was important previous medical record. Genital injuries were found only in 2 cases, contusions in an extra genital area in 2 cases too, and nonspecific erythema was found in 3 cases. All the patients collaborated with the physical examination and none had a physical disability as a consequence.

Conclusion: The most important aspect in a forensic legal evaluation is the expertise of the medical examiner who should be able to transmit this to stimulate the cooperation of the children to be evaluated.

\section{Referencias}

1. Laiioti SI, McClain N, Girardet R, McNeese M, Cheung K. Evaluating the Child for Sexual Abuse. Am Fam Physician. 2001, 63: 883-92.

2. Pollak S. Clinical forensic medicine and its main fields of activity from the foundation of the German Society of Legal Medicine until today. Forensic Sci Int. 2004; 144: 269-283.

3. Banaschak S y Brinkmann B. The role of clinical forensic medicine in cases of sexual child abuse. Forensic Sci Int. 1999; 99: 85-91.

4. Smith WG, Metcalfe M, Cormode EJ, Holder N. Approach to evaluation of sexual assault in children. Experience of a secondarylevel regional pediatric sexual assault clinic. Can Fam Physician. 2005, 51: 1347-1351.

5. Huyer D. Childhood sexual abuse and family physicians. Can Fam Physician. 2005, 51: 1317-1319.

6. Kellog $\mathrm{N}$ and the Committee on Child Abuse and Neglect. The evaluation of sexual abuse in children. Pediatrics 2005; 116: 506-512.

7. Christian CW, Lavelle JM, DeJong AR, Louiselle J, Brenner L, Joffe $\mathrm{M}$. Forensic evidence findings in prepuberal victims of sexual assault. Pediatrics 2000; 106: 100-104.

8. Pfeiffer L, Salvagni EP. Current view of sexual abuse in childhood and adolescence. J Pediatr (Río J). 2005; 81 (5 Suppl): S197-S204.

9. Hetznecker WH, Forman MA y Daruna JH. La entrevista clínica (anamnesis) . En: Behrman RE, Kliegman RM y Jenson HB. Nelson. Tratado de Pediatría. 16 edición. Mc Graw Hill Interamericana: México, 2001. 70-73.

10. Díaz A, Edwards S, Neal WP, Ludmer P, Sondike SB, Kessler C, Medeiros D, Nucci AT. Obtaining a history of sexual victimization from adolescent females seeking routine health care. Mt Sinai J Med 2004; 71: 170-172.

11. Gentile-Ramos I. Historia clínica. En: Gentile-Ramos Iy colaboradores. Semiología pediátrica. Segunda edición. Mc Graw Hill: Chile, 2000. 11-24.

12. Gisbert MS y Murcia Saiz E. Traumatismos en la infancia. En: Hernández Cueto, C. Valoración médica del daño corporal. Guía práctica para la exploración y evaluación de lesionados. Segunda edición. Masson SA: Barcelona, 2001. 371-389.

13. McGregor MJ, Le G, Marion SA, Wiebe E. Examination for sexual assault: Is the documentation of physical injury associated with the laying of charges? A retrospective cohort study. CMAJ 1999; 160: 1565-1569.

14. El mundo del niño: ¿Cómo lo descubrimos?. En: Papalia DE, Olds SW y Feldman RD. Psicología del Desarrollo de la infancia a la adolescencia. Novena edición. Mc Graw Hill: México, 2005. 20-50. 